MATHEMATICS OF COMPUTATION

Volume 66, Number 218, April 1997, Pages 807-822

S 0025-5718(97)00797-7

\title{
BOUNDS FOR MULTIPLICATIVE COSETS OVER FIELDS OF PRIME ORDER
}

\author{
COREY POWELL
}

\begin{abstract}
Let $m$ be a positive integer and suppose that $p$ is an odd prime with $p \equiv 1 \bmod m$. Suppose that $a \in(\mathbb{Z} / p \mathbb{Z})^{*}$ and consider the polynomial $x^{m}-a$. If this polynomial has any roots in $(\mathbb{Z} / p \mathbb{Z})^{*}$, where the coset representatives for $\mathbb{Z} / p \mathbb{Z}$ are taken to be all integers $u$ with $|u|<p / 2$, then these roots will form a coset of the multiplicative subgroup $\mu_{m}$ of $(\mathbb{Z} / p \mathbb{Z})^{*}$ consisting of the $m$ th roots of unity mod $p$. Let $C$ be a coset of $\mu_{m}$ in $(\mathbb{Z} / p \mathbb{Z})^{*}$, and define $|C|=\max _{u \in C}|u|$. In the paper "Numbers Having $m$ Small $m$ th Roots mod p" (Mathematics of Computation, Vol. 61, No. 203 (1993),pp. 393-413), Robinson gives upper bounds for $M_{1}(m, p)=\min _{C \in(\mathbb{Z} / p \mathbb{Z})^{*} / \mu_{m}}|C|$ of the form $M_{1}(m, p)<K_{m} p^{1-1 / \phi(m)}$, where $\phi$ is the Euler phi-function. This paper gives lower bounds that are of the same form, and seeks to sharpen the constants in the upper bounds of Robinson. The upper bounds of Robinson are proven to be optimal when $m$ is a power of 2 or when $m=6$.
\end{abstract}

\section{INTRODUCTION}

Let $\mathbb{Z}, \mathbb{Q}, \mathbb{R}$, and $\mathbb{C}$ denote the integers, rationals, real numbers, and complex numbers, respectively. Suppose that $m>1$ is a positive integer and that $p$ is an odd prime with $p \equiv 1 \bmod m$. Take the coset representatives for $\mathbb{Z} / p \mathbb{Z}$ to be all integers $u$ with $|u|<p / 2$. The multiplicative group $(\mathbb{Z} / p \mathbb{Z})^{*}$ has a subgroup $\mu_{m}$ of $m$ th roots of unity $\bmod p$, which is generated by a single element $t$.

If $a \in(\mathbb{Z} / p \mathbb{Z})^{*}$ has any $m$ th roots $\bmod p$, then these roots will form a coset of $\mu_{m}$ in $(\mathbb{Z} / p \mathbb{Z})^{*}$. Let $C$ be a coset of $\mu_{m}$. Define $|C|=\max _{u \in C}|u|$ and let $\|C\|=\sqrt{\sum_{u \epsilon C} u^{2}}$. These two measures of the "size" of $C$ are related by the inequality $\|C\| / \sqrt{m} \leq|C| \leq\|C\|$. Define $M_{1}(m, p)=\min _{C \in(\mathbb{Z} / p \mathbb{Z})^{*} / \mu_{m}}|C|$ and let $M_{2}(m, p)=\min _{C \in(\mathbb{Z} / p \mathbb{Z})^{*} / \mu_{m}}\|C\|$.

Let $K_{m}$ be the infimum of all $K$ 's such that $M_{1}(m, p) \leq K p^{1-1 / \phi(m)}$ for all $p \equiv 1 \bmod m$, where $\phi$ is the Euler phi-function. In [6], Robinson proves that such a $K_{m}$ exists, and gives the following upper bounds for $K_{m}$ :

1. $K_{m} \leq 2^{\tau}$, where $\tau$ is the number of distinct odd primes dividing $m$.

2. $K_{m} \leq 3$ if $m$ is divisible by only one prime greater than 3 .

3. $K_{m} \leq 2 / \sqrt{3}$ if $m$ is divisible by no prime greater than 3 .

Robinson conjectures that there are lower bounds for $M_{1}(m, p)$ of the form $M_{1}(m, p)$ $\geq K p^{1-1 / \phi(m)}$, but does not prove this result, and does not establish whether or not the upper bounds he gives for $K_{m}$ can be improved in general. In [3], Konyagin

Received by the editor May 30, 1995 and, in revised form, January 26, 1996.

1991 Mathematics Subject Classification. Primary 11A07, 11A15; Secondary 11N05, 11R18, $11 \mathrm{R} 44$.

(C) 1997 American Mathematical Society 
and Shparlinksi prove the lower bound $M_{1}(m, p)>(p-1) / 2-p^{3 / 2} / m$, which is a good bound if $p$ is small compared to $m$. Section 2 establishes that

$$
M_{1}(m, p) \geq\left(\sqrt{\phi(m)}\left(\prod_{\substack{q \text { prime } \\ q \mid m}} q^{1 /(q-1)}\right) / m\right) p^{1-1 / \phi(m)}
$$

if $p$ is sufficiently large compared to $m$. It follows from the bound above that

$$
M_{1}(m, p) \geq\left(m^{1 /(m-1)-1} \sqrt{\phi(m)}\right) p^{1-1 / \phi(m)},
$$

since $f(x)=x^{1 /(x-1)}$ is a decreasing function of $x$ for $x>1$. Section 2 proves that:

1. $K_{m} \leq \prod_{\substack{q \text { odd prime } \\ q \mid m}} q^{\frac{1}{2 q-2}}$, and

2. $K_{m} \leq 2 / \sqrt{3}$ if $m$ is divisible by no prime greater than 3 .

These upper bounds are at least as sharp as Robinson's for all $m$ and $p$. The first upper bound gives the estimates $K_{m}<C_{\epsilon} m^{\epsilon}$ for any $\epsilon>0$, where $C_{\epsilon}=$ $\prod_{\substack{q \text { odd prime } \\ 1 /(2 q-2)>\epsilon}} q^{1 /(2 q-2)-\epsilon}$. Hendrik Lenstra has suggested that $K_{m}<C \sqrt{\ln m}$ for some constant $C$, but this bound seems difficult to prove.

Section 9 discusses the possibility of improving these upper and lower bounds.

\section{LOWER BOUNDS FOR $M_{1}(m, p)$ AND $M_{2}(m, p)$}

Let $\zeta_{m}$ be a primitive $m$ th root of unity. It is well known from Galois theory that $\mathbb{Q}\left(\zeta_{m}\right)$ is a Galois extension of $\mathbb{Q}$ of degree $\phi(m)$, and that the elements $\sigma_{j}$ of the Galois group $\operatorname{Gal}\left(\mathbb{Q}\left(\zeta_{m}\right) / \mathbb{Q}\right)$ of $\mathbb{Q}\left(\zeta_{m}\right)$ over $\mathbb{Q}$ are uniquely defined by the condition $\sigma_{j}\left(\zeta_{m}\right)=\zeta_{m}^{j}$, where $\operatorname{gcd}(j, m)=1$. Let $N_{\mathbb{Q}\left(\zeta_{m}\right) / \mathbb{Q}}()$ and $\operatorname{Tr}_{\mathbb{Q}\left(\zeta_{m}\right) / \mathbb{Q}}()$ denote the norm and trace maps from $\mathbb{Q}\left(\zeta_{m}\right)$ to $\mathbb{Q}$. It is well known from algebraic number theory that the irreducible polynomial of $\zeta_{m}$ over $\mathbb{Q}$ is the $m$ th cyclotomic polynomial $\Phi_{m}(X)=\prod_{i \in(\mathbb{Z} / m \mathbb{Z})^{*}}\left(X-\zeta_{m}^{i}\right)$, and that the ring of integers of $\mathbb{Q}\left(\zeta_{m}\right)$ is $\mathbb{Z}\left[\zeta_{m}\right]$. The ideal generated by $p$ in $\mathbb{Z}\left[\zeta_{m}\right]$ factors as $p \mathbb{Z}\left[\zeta_{m}\right]=\prod_{i \in(\mathbb{Z} / m \mathbb{Z})^{*}} P_{i}$, where

$$
P_{i}=p \mathbb{Z}\left[\zeta_{m}\right]+\left(\zeta_{m}-t^{i}\right) \mathbb{Z}\left[\zeta_{m}\right]
$$

The following theorem will also use the facts that $N_{\mathbb{Q}\left(\zeta_{m}\right) / \mathbb{Q}}\left(P_{i}\right)=p$ and that $P_{i} \cap \mathbb{Z}=p \mathbb{Z}$.

Let $l$ be the largest prime dividing $m$ such that $M_{1}(m, p)<p / l$, if such a prime exists, and let $l=1$ otherwise. If $p>\left(2^{\tau} \max _{q \mid m, q \text { prime }} q\right)^{\phi(m)}$, then $M_{1}(m, p)<$ $2^{\tau} p^{1-1 / \phi(m)}<p / \max _{q \mid m, q \text { prime }} q$ by the results of Robinson, and so $l$ will be the largest prime dividing $m$.

Theorem 1. If $l$ is as above, then

$$
M_{1}(m, p) \geq\left(\sqrt{\phi(m)}\left(\prod_{q \text { prime, } q \leq l} q^{\frac{1}{q-1}}\right) / m\right) p^{1-1 / \phi(m)} .
$$

The proof of the theorem will follow directly from the following three lemmas together with the fact that $\left|N_{\mathbb{Q}\left(\zeta_{m}\right) / \mathbb{Q}} \alpha\right|=N_{\mathbb{Q}\left(\zeta_{m}\right) / \mathbb{Q}}\left(\alpha \mathbb{Z}\left[\zeta_{m}\right]\right)$ for any $\alpha \in \mathbb{Z}\left[\zeta_{m}\right]$. Let $C$ be a coset of $(\mathbb{Z} / p \mathbb{Z})^{*}$, and let $b_{0}, \ldots, b_{m-1}$ be the elements of $C$ with $b_{j} \equiv b_{0} t^{j} \bmod p$. Define $\beta_{d}=\sum_{j=0}^{m-1} b_{j} \zeta_{m}^{j d}$, and let $\bar{\beta}$ denote the complex conjugate of $\beta$.

Lemma 1.1. If $\beta_{1}$ is as above, and $C$ is such that $|C|=M_{1}(m, p)$, then $q^{\frac{\phi(m)}{q-1}} \mid$ $N_{\mathbb{Q}\left(\zeta_{m}\right) / \mathbb{Q}}\left(\beta_{1}\right)$ for all $q \leq l$. 
Proof. It suffices to show that $\beta_{1} \in\left(\zeta_{m}^{m / q}-1\right) \mathbb{Z}\left[\zeta_{m}\right]$, since

$$
N_{\mathbb{Q}\left(\zeta_{m}\right) / \mathbb{Q}}\left(\zeta_{m}^{m / q}-1\right)=N_{\mathbb{Q}\left(\zeta_{q}\right) / \mathbb{Q}}\left(N_{\mathbb{Q}\left(\zeta_{m}\right) / \mathbb{Q}\left(\zeta_{q}\right)}\left(\zeta_{m}^{m / q}-1\right)\right)=q^{\frac{\phi(m)}{q-1}} .
$$

It is clear that $\zeta_{m}^{i} \equiv \zeta_{m}^{i \bmod (m / q)} \bmod \left(\zeta_{m}^{m / q}-1\right) \mathbb{Z}\left[\zeta_{m}\right]$, and hence that

$$
\beta_{1} \equiv \sum_{j=0}^{\frac{m}{q}-1}\left(\zeta_{m}^{j} \sum_{k=0}^{q-1} b_{j+k m / q}\right) \bmod \left(\zeta_{m}^{m / q}-1\right) \mathbb{Z}\left[\zeta_{m}\right] .
$$

It follows from the definition of $b_{i}$ that

$$
\begin{aligned}
\sum_{k=0}^{q-1} b_{j+k m / q} & \equiv b_{j} \sum_{k=0}^{q-1} t^{k m / q} \bmod p \\
& \equiv b_{j} \frac{1-t^{m}}{1-t^{m / q}} \bmod p \\
& \equiv 0 \bmod p
\end{aligned}
$$

for $0 \leq j \leq \frac{m}{q}-1$. It now follows that $\sum_{k=0}^{q-1} b_{j+k m / q}=0$ for $0 \leq j \leq \frac{m}{q}-1$ because

$$
\left|\sum_{k=0}^{q-1} b_{j+k m / q}\right| \leq \sum_{k=0}^{q-1}\left|b_{j+k m / q}\right|<p q / l \leq p .
$$

This proves the lemma.

Lemma 1.2. If $\beta_{1}$ is as above, then $\beta_{1} \neq 0$, and

$$
p^{2(\phi(m)-1)} \mid N_{\mathbb{Q}\left(\zeta_{m}\right) / \mathbb{Q}}\left(\beta_{1} \overline{\beta_{1}}\right) .
$$

Proof. It follows from the definition of $P_{j}$ that $\zeta_{m} \equiv t^{j} \bmod P_{j}$, where $\operatorname{gcd}(j, m)=1$, and hence $\beta_{1} \equiv b_{0} \sum_{k=0}^{m-1} t^{k(j+1)} \bmod P_{j}$. This sum is a geometric series, and so $\beta_{1} \equiv b_{0}\left(1-t^{m(j+1)}\right)\left(1-t^{j+1}\right)^{-1} \equiv 0 \bmod P_{j}$ provided that $j \neq m-1$. It follows that $p^{\phi(m)-1} \mid N_{\mathbb{Q}\left(\zeta_{m}\right) / \mathbb{Q}}\left(\beta_{1}\right)$. If $j=m-1$, then $\beta_{1} \equiv b_{0} m \not \equiv 0 \bmod P_{j}$, which implies that $\beta_{1} \notin P_{m-1}$ and hence that $\beta_{1} \neq 0$. The lemma now follows since $N_{\mathbb{Q}\left(\zeta_{m}\right) / \mathbb{Q}}\left(\beta_{1}\right)=N_{\mathbb{Q}\left(\zeta_{m}\right) / \mathbb{Q}}\left(\overline{\beta_{1}}\right)$.

It is a direct consequence of Lemma 1.1 and Lemma 1.2 that

$$
\left|N_{\mathbb{Q}\left(\zeta_{m}\right) / \mathbb{Q}}\left(\beta_{1} \overline{\beta_{1}}\right)\right| \geq\left(\prod_{q \text { prime, } q \leq l} q^{2 \phi(m) / p-1}\right) p^{2(\phi(m)-1)}
$$

if $|C|=M_{1}(m, p)$. The theorem will now follow from taking the $2 \phi(m)$ th root of this inequality and combining it with the following inequality.

Lemma 1.3. If $\beta_{1}$ is as above, then

$$
|C| \geq(\sqrt{\phi(m)} / m)\left|N_{\mathbb{Q}\left(\zeta_{m}\right) / \mathbb{Q}}\left(\beta_{1} \overline{\beta_{1}}\right)\right|^{1 /(2 \phi(m))} .
$$

Proof. It follows from the arithmetic-geometric mean inequality that

$$
\operatorname{Tr}_{\mathbb{Q}\left(\zeta_{m}\right) / \mathbb{Q}}\left(\beta_{1} \overline{\beta_{1}}\right) / m^{2} \geq\left(\phi(m) / m^{2}\right)\left(N_{\mathbb{Q}\left(\zeta_{m}\right) / \mathbb{Q}}\left(\beta_{1} \overline{\beta_{1}}\right)\right)^{1 / \phi(m)}
$$

The lemma follows by combining this inequality with the following lemma and the inequality $|C|^{2} \geq\|C\|^{2} / m$ and then taking the square root of both sides. 
Lemma 1.4. If $\beta_{1}$ is as above, then

$$
m\|C\|^{2} \geq \operatorname{Tr}_{\mathbb{Q}\left(\zeta_{m}\right) / \mathbb{Q}}\left(\beta_{1} \overline{\beta_{1}}\right)
$$

where equality holds if $\beta_{d}=0$ for all $d$ with $\operatorname{gcd}(d, m) \neq 1$.

Proof. The lemma is a consequence of the following computation.

$$
\begin{aligned}
\operatorname{Tr}_{\mathbb{Q}\left(\zeta_{m}\right) / \mathbb{Q}}\left(\beta_{1} \overline{\beta_{1}}\right) & =\sum_{j \in(\mathbb{Z} / m \mathbb{Z})^{*}}\left(\sum_{k=0}^{m-1} b_{k} \zeta_{m}^{j k}\right)\left(\sum_{k=0}^{\overline{m-1} b_{k} \zeta_{m}^{j k}}\right) \\
& \leq \sum_{j \bmod m}\left(\sum_{k=0}^{m-1} b_{k} \zeta_{m}^{j k}\right)\left(\sum_{k=0}^{m-1} b_{k} \zeta_{m}^{j k}\right) \\
& =\sum_{j \bmod m} \sum_{k=0}^{m-1} \sum_{l=0}^{m-1} b_{k} b_{l} \zeta_{m}^{(k-l) j} \\
& =m\|C\|^{2}+\sum_{0 \leq k, l \leq m-1} b_{k} b_{l} \sum_{j \neq l} \zeta_{m}^{(k-l) j} \\
& =m\|C\|^{2} .
\end{aligned}
$$

Combining the direct consequence of Lemma 1.1 and Lemma 1.2 with the arithmetic-geometric mean inequality and Lemma 1.4 gives the following lower bound for $M_{2}(m, p)$.

Theorem 2. If $M_{2}(m, p)$ is as previously defined, then

$$
M_{2}(m, p) \geq\left(\sqrt{\phi(m) / m} \prod_{\substack{q \text { prime }, \leq l \\ q \mid m}} q^{\frac{1}{q-1}}\right) p^{1-1 / \phi(m)} .
$$

If $p$ is sufficiently large compared to $m$, then

$$
M_{2}(m, p) \geq\left(m^{1 /(m-1)} \sqrt{\phi(m) / m}\right) p^{1-1 / \phi(m)} .
$$

An upper bound for this measure will be given in Section 2 .

$$
\text { 2. UPPER BOUNDS FOR } M_{1}(m, p) \text { AND } M_{2}(m, p)
$$

The following upper bounds are obtained by using Minkowski's geometry of numbers. The first upper bound below also gives the estimate $M_{1}(m, p)<$ $C_{\epsilon} m^{\epsilon} p^{1-1 / \phi(m)}$ for any $\epsilon>0$, where $C_{\epsilon}$ is as defined in Section 1.

Theorem 3. If $m$ and $p$ are as above, then

$$
M_{1}(m, p) \leq \min \left(p^{1-1 / m},\left(\prod_{\substack{q \text { odd prime } \\ q \mid m}} q^{1 /(2 q-2)}\right) p^{1-1 / \phi(m)}\right) .
$$

If 3 is the only odd prime dividing $m$, then

$$
M_{1}(m, p) \leq \min \left(p^{1-1 / m},(2 / \sqrt{3}) p^{1-1 / \phi(m)}\right) .
$$

If $\Lambda$ is a lattice of full rank in $\mathbb{R}^{n}$ and $B=\left\{v_{i}\right\}_{i=1}^{n}$ is an ordered $\mathbb{Z}$-basis for $\Lambda$, then let $d(\Lambda)=|\operatorname{det}(A)|$, where the $i$ th column of $A$ is $v_{i}$. This determinant is independent of the choice of ordered basis for $\Lambda$. Note that $d(\Lambda)=\sqrt{|\operatorname{det}(M)|}$, where $M_{i j}=\left\langle v_{i}, v_{j}\right\rangle$ and $\langle$,$\rangle is the standard Euclidean inner product. The$ theorem above is a consequence of the following theorem (see [5], p. 120). 
Theorem 4. Let $\Lambda$ be a lattice (of full rank) in $\mathbb{R}^{n}$ and let $K$ be a bounded 0symmetric convex body of volume $\operatorname{vol}(K)>2^{n} d(\Lambda)$. Then $K$ contains a point $x \neq 0$ of $\Lambda$.

Let $\Psi_{m}(X)$ be the $m-\phi(m)$ th degree polynomial $\left(X^{m}-1\right) / \Phi_{m}(X)$ and define

$$
V=\left\{\left(b_{0}, \ldots, b_{m-1}\right) \in \mathbb{R}^{m} \mid \sum_{j=0}^{m-1} b_{j} X^{j}=\Psi_{m}(X) \Theta(X), \Theta(X) \in \mathbb{R}[X]\right\} .
$$

The subspace $V$ is $\phi(m)$-dimensional since $V$ is isomorphic to the subspace of $\mathbb{R}[X]$ consisting of polynomials $r$ such that $\Psi_{m}(X) \mid r$ and $\operatorname{deg}(r)<m$. This subspace has a basis $\beta=\left\{\Psi_{m}(X), X \Psi_{m}(X), \ldots, X^{\phi(m)-1} \Psi_{m}(X)\right\}$. The vector space $V$ contains the lattice

$$
L=\left\{\left(b_{0}, \ldots, b_{m-1}\right) \in \mathbb{Z}^{m} \mid \sum_{j=0}^{m-1} b_{j} X^{j}=\Psi_{m}(X) \Theta(X), \Theta(X) \in \mathbb{Z}[X]\right\} .
$$

Define also the lattice

$$
\mathcal{C}=\left\{\left(b_{0}, \ldots, b_{m-1}\right) \in \mathbb{Z}^{m} \mid b_{j} \equiv b_{0} t^{j} \bmod p, 0 \leq j \leq m-1\right\}
$$

in $\mathbb{R}^{m}$, and let

$$
S_{r}=\left\{\left(b_{0}, \ldots, b_{m-1}\right) \in \mathbb{R}^{m}\left|\max _{0 \leq j \leq m-1}\right| b_{j} \mid<r\right\} .
$$

If $\left(b_{0}, \ldots, b_{m-1}\right) \in \mathcal{C}$ and $b_{0} \not \equiv 0 \bmod p$, then there is a coset $C$ such that $C=$ $\left\{b_{i} \bmod p \mid 0 \leq i \leq m-1\right\}$ and so

$$
M_{1}(m, p) \leq|C| \leq \max _{0 \leq j \leq m-1}\left|b_{j}\right| .
$$

If $r$ can be chosen so that $r<p$, then $\left(b_{0}, \ldots, b_{\phi(m)-1}\right) \in S_{r} \cap(\mathcal{C} \cap L)$ will have $b_{0} \not \equiv 0 \bmod p$. The following lemma proves the first part of Theorem 3.

Lemma 4.1. If $m$ and $p$ are as above, then $M_{1}(m, p) \leq p^{1-1 / m}$.

Proof. Let $e_{i}$ be the $i$ th standard basis element in $\mathbb{R}^{m}$. The set $B=\left\{\left(1, t, \ldots, t^{m-1}\right)\right.$, $\left.p e_{i} \mid 2 \leq i \leq m\right\}$ forms a $\mathbb{Z}$-basis for $\mathcal{C}$, and hence $d(\mathcal{C})=p^{m-1}$. It is now clear from Theorem 4 that $S_{r}$ will contain a point of $\mathcal{C}$ if $(2 r)^{m}>2^{m} p^{m-1}$, or if $r>p^{1-1 / m}$. The lemma now follows from the earlier remarks.

Now, suppose that

$$
p^{1-1 / m}>\left(\prod_{\substack{q \text { odd prime } \\ q \mid m}} q^{1 /(2 q-2)}\right) p^{1-1 / \phi(m)} .
$$

To apply Theorem 4, define $d(L)=d(I(L))$, where $I$ is an isometry from $V$ to $\mathbb{R}^{\phi(m)}$. Note that this definition is independent of the choice of $I$ and that $d(L)=$ $\sqrt{|\operatorname{det}(M)|}$, where $M_{i j}=\left\langle v_{i}, v_{j}\right\rangle$ and $B=\left\{v_{i}\right\}_{i=1}^{n}$ is a basis for $L$ as a $\mathbb{Z}$-module. If the $\phi(m)$-dimensional volume $\operatorname{vol}\left(S_{r} \cap V\right)>2^{\phi(m)} d(\mathcal{C} \cap L)$, then Theorem 4 would imply that there is a non-zero point of $\mathcal{C} \cap L$ in $S_{r} \cap V$. It would then be a consequence of these remarks together with the following theorem that there is a non-zero point of $\mathcal{C} \cap L$ in $S_{r} \cap V$ if

1. $r>(d(\mathcal{C} \cap L))^{1 / \phi(m)}$,

2. $r>(d(\mathcal{C} \cap L))^{1 / \phi(m)} / \sqrt{2}$ for $m$ even, and

3. $r>((2 / \sqrt{3}) d(\mathcal{C} \cap L) / d(L))^{1 / \phi(m)}$ if 3 is the only odd prime dividing $m$. 
If $r$ can be chosen such that $r<p$, then it would follow that $M_{1}(m, p) \leq r$. The following two theorems will prove Theorem 3. Note that $M_{1}(m, p)=M_{1}(2 m, p)$ if $m$ is odd, since the roots of $X^{2 m}-u \bmod p$ are of the form $\pm x$, where $x$ is a root of $X^{m}-z$ and $z^{2} \equiv u \bmod p$.

Theorem 5. The following are lower bounds for $\operatorname{vol}\left(S_{r} \cap V\right)$ :

1. $\operatorname{vol}\left(S_{r} \cap V\right) \geq(2 r)^{\phi(m)}$,

2. $\operatorname{vol}\left(S_{r} \cap V\right) \geq(2 \sqrt{2} r)^{\phi(m)}$ if $m$ is even,

3. $\operatorname{vol}\left(S_{r} \cap V\right)=(\sqrt{3} r)^{\phi(m)} d(L)$ if $m=2^{e} 3^{f}$, with $e, f>0$.

Proof. A result of Vaaler (see [8]) shows that $\operatorname{vol}\left(S_{1 / 2} \cap V\right) \geq 1$. A change of variables then establishes the first lower bound. To prove the second lower bound, define

$$
W=\left\{\left(b_{0}, \ldots b_{m-1}\right) \mid \sum_{j=0}^{m-1} b_{j} X^{j}=\left(X^{m / 2}-1\right) \Theta(X), \Theta(X) \in \mathbb{R}[X]\right\} .
$$

The fact that $m$ is even implies that $X^{m / 2}-1 \mid \Psi_{m}(X)$, and so $V \subset W$. The set $B_{W}=\left\{w_{i}\right\}_{i=0}^{m / 2-1}$ is an ordered orthogonal basis for $W$, where $w_{i}$ has -1 in the $i$ th coordinate, 1 in the $(i+m / 2)$ th coordinate, and 0 in all other coordinates. It follows that $w \in S_{r} \cap W$ if and only if $\left|a_{i}\right|<r$ for $0 \leq i \leq m / 2-1$, where $a_{i}$ is the $i$ th coordinate of $w$ with respect to the basis $B_{W}$. Map $S_{r} \cap W$ isometrically to the box $S_{r \sqrt{2}}$ in $\mathbb{R}^{m / 2}$ by taking $w_{i}$ to $\sqrt{2} e_{i+1}$. Applying the result of Vaaler and a change of variables then shows that

$$
\begin{aligned}
\operatorname{vol}\left(S_{r} \cap V\right) & =\operatorname{vol}\left(\left(S_{r} \cap W\right) \cap V\right) \\
& =\operatorname{vol}\left(S_{r \sqrt{2}} \cap V^{\prime}\right) \\
& =(2 \sqrt{2} r)^{\phi(m)} \operatorname{vol}\left(S_{1 / 2} \cap V^{\prime}\right) \\
& \geq(2 \sqrt{2} r)^{\phi(m)},
\end{aligned}
$$

where $V^{\prime}$ is the image of $V$ in $\mathbb{R}^{m / 2}$. This establishes the second lower bound.

If $m=2^{e} 3^{f}$ with $e, f>0$, then

$$
X^{m}-1=\left(X^{m / 2}-1\right)\left(X^{m / 6}+1\right)\left(X^{m / 3}-X^{m / 6}+1\right),
$$

with $\Phi_{m}(X)=X^{m / 3}-X^{m / 6}+1$, and hence $\Psi_{m}(X)=\left(X^{m / 2}-1\right)\left(X^{m / 6}+1\right)$. The set $B=\left\{w_{i}+w_{i+m / 6}\right\}_{i=0}^{m / 3-1}$ is an ordered basis for $V$, and so $B^{\prime}=$ $\left\{\sqrt{2}\left(e_{i}+e_{i+m / 6}\right)\right\}_{i=1}^{m / 3}$ forms an ordered basis for $V^{\prime}$. If $a_{i}$ denotes the $i$ th coordinate of $v^{\prime} \in V^{\prime}$ with respect to the basis $B^{\prime}$, then the $i$ th coordinate of $v^{\prime}$ with respect to the standard basis is

1. $\sqrt{2} a_{i}$ if $1 \leq i \leq m / 6$,

2. $\sqrt{2}\left(a_{i}+a_{i-m / 6}\right)$ if $m / 6<i \leq m / 3$, and

3. $\sqrt{2} a_{i-m / 6}$ if $m / 3<i \leq m / 2$.

Hence $v^{\prime} \in S_{1 / 2} \cap V^{\prime}$ if and only if $\left|a_{i}\right|<\frac{1}{2 \sqrt{2}}$ for $1 \leq i \leq m / 3$ and $\left|a_{i}+a_{i+m / 6}\right|<$ $\frac{1}{2 \sqrt{2}}$ for $1 \leq i \leq m / 6$. The computation at the end of the proof of the second lower bound proved that

$$
\operatorname{vol}\left(S_{r} \cap V\right)=(2 \sqrt{2} r)^{\phi(m)} \operatorname{vol}\left(S_{1 / 2} \cap V^{\prime}\right) .
$$


Map $V^{\prime}$ to $\mathbb{R}^{m / 3}$ by taking $\sqrt{2}\left(e_{i}+e_{i+m / 6}\right)$ to $e_{i}$ in $\mathbb{R}^{m / 3}$. The volume of the image of $S_{1 / 2} \cap V^{\prime}$ in $\mathbb{R}^{m / 3}$ can be found by evaluating the multiple integral

$$
\int_{\frac{-1}{2 \sqrt{2}}}^{\frac{1}{2 \sqrt{2}}} \cdots \int_{\frac{-1}{2 \sqrt{2}}}^{\frac{1}{2 \sqrt{2}}} \int_{\max \left(\frac{-1}{2 \sqrt{2}}, \frac{-1}{2 \sqrt{2}}-x_{1}\right)}^{\min \left(\frac{1}{2 \sqrt{2}}, \frac{1}{2 \sqrt{2}}-x_{1}\right)} \cdots \int_{\max \left(\frac{-1}{2 \sqrt{2}}, \frac{-1}{2 \sqrt{2}}-x_{m / 6}\right)}^{\min \left(\frac{1}{2 \sqrt{2}}, \frac{1}{2 \sqrt{2}}-x_{m / 6}\right)} d x_{m / 3} \cdots d x_{1},
$$

which has the same value as

$$
\left(\int_{-1 /(2 \sqrt{2})}^{1 /(2 \sqrt{2})} \int_{\max \left(-1 /(2 \sqrt{2}),-1 /(2 \sqrt{2})-x_{1}\right)}^{\min \left(1 /(2 \sqrt{2}), 1 /(2 \sqrt{2})-x_{1}\right)} d x_{2} d x_{1}\right)^{m / 6} .
$$

A routine computation shows that the value of this double integral is $3 / 8$. It follows that $\operatorname{vol}\left(S_{1 / 2} \cap V^{\prime}\right)=(3 / 8)^{m / 6} d(L)$ since $B$ is a $\mathbb{Z}$-basis for $L$ which maps isometrically to $B^{\prime}$. Substituting this value for $S_{1 / 2} \cap V^{\prime}$ into (1) proves the third equation, which finishes the proof of the theorem.

Theorem 6. If $\mathcal{C}$ and $L$ are as above, then

$$
d(\mathcal{C} \cap L)=\left(\prod_{q \text { prime }} q^{\phi \mid m} \underset{ }{\phi(m) /(2 q-2)}\right) p^{\phi(m)-1} .
$$

The theorem will be proven by a sequence of lemmas that reduce the theorem to problems in algebraic number theory. The following lemma reduces finding $d(\mathcal{C} \cap L)$ to finding $d(L)$.

Lemma 6.1. If $\mathcal{C}$ and $L$ are as above, then $d(\mathcal{C} \cap L)=p^{\phi(m)-1} d(L)$.

Proof. It suffices to show that $\#(L /(\mathcal{C} \cap L))=p^{\phi(m)-1}$, since

$$
d(\mathcal{C} \cap L) / d(L)=\#(L /(\mathcal{C} \cap L)) .
$$

There is a homomorphism $\Omega$ from $L$ to $\mathbb{Z}\left[\zeta_{m}\right]$ defined by $\Omega\left(b_{0}, \ldots, b_{m-1}\right)=$ $\sum_{j=0}^{m-1} b_{j} \zeta_{m}^{j}$, with $\Omega(L)=\Psi_{m}\left(\zeta_{m}\right) \mathbb{Z}\left[\zeta_{m}\right]$. If $\Omega\left(l_{0}, \ldots, l_{m-1}\right)=0$, then

$$
\Phi_{m}(X) \Psi_{m}(X) \mid \sum_{j=0}^{m-1} l_{j} X^{j},
$$

and so $\left(l_{0}, \ldots, l_{m-1}\right)=0$ since $\Phi_{m}(X) \Psi_{m}(X)=X^{m}-1$. This shows that $\Omega$ is injective. The set

$$
\mathcal{B}=\left\{\zeta_{m}^{j} \Psi_{m}\left(\zeta_{m}\right) \mid 0 \leq j \leq \phi(m)-1\right\}
$$

forms a basis for $\Psi_{m}\left(\zeta_{m}\right) \mathbb{Z}\left[\zeta_{m}\right]$ as a $\mathbb{Z}$-module, and so $\Omega^{-1}(\mathcal{B})$ forms a basis for $L$ as a $\mathbb{Z}$-module. To determine $\#(L /(\mathcal{C} \cap L))$, consider the $\mathbb{Z} / p \mathbb{Z}$ vector spaces $L / p L$ and $(\mathcal{C} \cap L) / p L$. The projection of $\Omega^{-1}(\mathcal{B})$ to $L / p L$ will form a basis for $L / p L$, and so $L / p L$ is $\phi(m)$-dimensional.

If $r \in \mathbb{Z}[X]$, then let $\rho(r)$ denote the polynomial in $\mathbb{Z} / p \mathbb{Z}[X]$ derived by reducing the coefficients of $r \bmod p$. The proof of Lemma 1.2 demonstrated that $\left(X-t^{j}\right) \mid$ $\rho\left(\sum_{k=0}^{m-1} t^{k} X^{k}\right)$ if $j \not \equiv-1 \bmod p$ and hence that $\rho\left(\Psi_{m}(X)\right) \mid \rho\left(\sum_{k=0}^{m-1} t^{k} X^{k}\right)$. Let $\Upsilon(X)=h(X) \Psi_{m}(X)$, where

$$
\rho\left(h(X) \Psi_{m}(X)\right)=\rho\left(\sum_{j=0}^{m-1} t^{j} X^{j}\right),
$$


and write the coefficients of $\Upsilon(X)$ into a vector $v \in \mathbb{Z}^{m}$. From the construction of $v$, it is clear that $v \in L \cap \mathcal{C}$ and that $v \notin p L$. From the definition of $\mathcal{C}$, it follows that $v$ spans $(L \cap \mathcal{C}) / p L$ and hence that

$$
\#\{L /(L \cap \mathcal{C})\}=\#\{(L / p L) /((L \cap \mathcal{C}) / p L)\}=p^{\phi(m)-1} .
$$

This proves the lemma.

It is another consequence of the lemma that $d(L)=\sqrt{|\operatorname{det}(M)|}$, where

$$
M_{i j}=\left\langle\Omega^{-1}\left(\zeta_{m}^{i-1} \Psi_{m}\left(\zeta_{m}\right)\right), \Omega^{-1}\left(\zeta_{m}^{j-1} \Psi_{m}\left(\zeta_{m}\right)\right)\right\rangle .
$$

For $\alpha, \gamma \in \mathbb{Z}\left[\zeta_{m}\right]$, define $\langle\alpha, \gamma\rangle_{m}=\operatorname{Tr}_{\mathbb{Q}\left(\zeta_{m}\right) / \mathbb{Q}}(\alpha \bar{\gamma}) \in \mathbb{Q}$. The following lemma will be important in finding $|\operatorname{det}(M)|$.

Lemma 6.2. If $u, v \in L$, then $\langle u, v\rangle=\langle\Omega(u), \Omega(v)\rangle_{m} / m$.

Proof. It suffices to prove the lemma in the case $u=v$, since

$$
\langle u, v\rangle=(\langle u+v, u+v\rangle-\langle u-v, u-v\rangle) / 4
$$

and

$$
\langle\Omega(u), \Omega(v)\rangle_{m}=\left(\langle\Omega(u+v), \Omega(u+v)\rangle_{m}-\langle\Omega(u-v), \Omega(u-v)\rangle_{m}\right) / 4 .
$$

Let $u=\left(b_{0}, \ldots, b_{m-1}\right)$; it follows that $\sum_{k=0}^{m-1} b_{k} \zeta^{k d}=0$ for $\operatorname{gcd}(d, m) \neq 1$ because $u \in L$. The lemma now follows from Lemma 1.4.

It is a consequence of Lemma 6.2 that $\sqrt{|\operatorname{det}(M)|}=\sqrt{|\operatorname{det}(D)|} / m^{\phi(m) / 2}$, where $D_{j k}=\operatorname{Tr}_{\mathbb{Q}\left(\zeta_{m}\right) / \mathbb{Q}}\left(\zeta_{m}^{j} \Psi_{m}\left(\zeta_{m}\right) \overline{\zeta_{m}^{k} \Psi_{m}\left(\zeta_{m}\right)}\right)$. The next lemma gives the latter determinant in terms of the discriminant of $\Psi_{m}\left(\zeta_{m}\right) \mathbb{Z}\left[\zeta_{m}\right]$.

Lemma 6.3. If $D$ is as above, then $|\operatorname{det}(D)|=\left|D_{\mathbb{Q}\left(\zeta_{m}\right) / \mathbb{Q}}\left(\Psi_{m}\left(\zeta_{m}\right) \mathbb{Z}\left[\zeta_{m}\right]\right)\right|$, where $D_{\mathbb{Q}\left(\zeta_{m}\right) / \mathbb{Q}}()$ denotes the discriminant.

Proof. If $\operatorname{Gal}\left(\mathbb{Q}\left[\zeta_{m}\right] / \mathbb{Q}\right)=\left\{\sigma_{1}, \ldots, \sigma_{\phi(m)}\right\}$, then $D=P P^{*}$, where $P_{j k}=$ $\sigma_{k}\left(\zeta_{m}^{j} \Psi_{m}\left(\zeta_{m}\right)\right)$ and $P^{*}$ is the conjugate transpose of $P$. The determinant is a polynomial in its entries, and so the following calculation proves the lemma:

$$
\begin{aligned}
|\operatorname{det}(D)| & =\left|\operatorname{det}(P) \operatorname{det}\left(P^{*}\right)\right| \\
& =|\operatorname{det}(P) \overline{\operatorname{det}(P)}| \\
& =\left|(\operatorname{det}(P))^{2}\right| \\
& =\left|D_{\mathbb{Q}\left(\zeta_{m}\right) / \mathbb{Q}}\left(\Psi_{m}\left(\zeta_{m}\right) \mathbb{Z}\left[\zeta_{m}\right]\right)\right| .
\end{aligned}
$$

It is known from algebraic number theory (see [4], p. 66) that

$$
D_{\mathbb{Q}\left(\zeta_{m}\right) / \mathbb{Q}}\left(\Psi_{m}\left(\zeta_{m}\right) \mathbb{Z}\left[\zeta_{m}\right]\right)=\left(N_{\mathbb{Q}\left(\zeta_{m}\right) / \mathbb{Q}}\left(\Psi_{m}\left(\zeta_{m}\right)\right)\right)^{2} D_{\mathbb{Q}\left(\zeta_{m}\right) / \mathbb{Q}}\left(\mathbb{Z}\left[\zeta_{m}\right]\right) .
$$

Differentiating the equation $X^{m}-1=\Phi_{m}(X) \Psi_{m}(X)$ and substituting $\zeta_{m}$ for $X$ gives $m \zeta_{m}^{m-1}=\Phi_{m}^{\prime}\left(\zeta_{m}\right) \Psi_{m}\left(\zeta_{m}\right)$. Taking the norm of both sides gives

$$
m^{\phi(m)}=\left|D_{\mathbb{Q}\left(\zeta_{m}\right) / \mathbb{Q}}\left(\mathbb{Z}\left[\zeta_{m}\right]\right) N_{\mathbb{Q}\left(\zeta_{m}\right) / \mathbb{Q}}\left(\Psi_{m}\left(\zeta_{m}\right)\right)\right| .
$$

Hence $\operatorname{det}(D)=m^{2 \phi(m)} /\left|D_{\mathbb{Q}\left(\zeta_{m}\right) / \mathbb{Q}}\left(\mathbb{Z}\left[\zeta_{m}\right]\right)\right|$ and so

$$
\begin{aligned}
d(L) & =\sqrt{|\operatorname{det}(M)|} \\
& =\sqrt{|\operatorname{det}(D)|} / m^{\phi(m) / 2} \\
& =m^{\phi(m) / 2}\left|D_{\mathbb{Q}\left(\zeta_{m}\right) / \mathbb{Q}}\left(\mathbb{Z}\left[\zeta_{m}\right]\right)\right|^{-1 / 2} .
\end{aligned}
$$


It is also known from algebraic number theory (see [1], p. 88) that

$$
\left|D_{\mathbb{Q}\left(\zeta_{m}\right) / \mathbb{Q}}\left(\mathbb{Z}\left[\zeta_{m}\right]\right)\right|=m^{\phi(m)} / \prod_{q \text { prime }} q_{q \mid m} q^{\phi(m) /(q-1)} .
$$

This gives $d(L)=\prod_{\substack{q \mid m \\ \text { prime }}} q^{\phi(m) /(2 q-2)}$. Theorem 6 follows from Lemma 6.1 together with the above equality.

The inequality $M_{2}(m, p) \leq(\sqrt{m}) M_{1}(m, p)$ gives an upper bound for $M_{2}(m, p)$. If $p$ is sufficiently large compared to $m$, then this bound can be improved by considering spheres. Let $\mathcal{S}_{r}=\left\{x \in \mathbb{R}^{m} \mid \sqrt{\langle x, x\rangle}<r\right\}$. In this case, $\operatorname{vol}\left(\mathcal{S}_{r} \cap V\right)=$ $r^{\phi(m)} m^{\phi(m) / 2} / \Gamma(\phi(m) / 2+1)$, where $\Gamma$ is the gamma function. If

$$
r^{\phi(m)} m^{\phi(m) / 2} / \Gamma(\phi(m) / 2+1)>\left(2^{\phi(m)} \prod_{q \underset{q \mid m}{\operatorname{prime}}} q^{\phi(m) /(2 q-2)}\right) p^{\phi(m)-1},
$$

then there will be a non-zero point of $\mathcal{C} \cap L$ that is in $\mathcal{S}_{r} \cap V$ by Theorem 4 and Theorem 6. Solving for $r$ gives

$$
r>\left(\frac{2(\Gamma(\phi(m) / 2+1))^{1 / \phi(m)} \prod_{q \text { prime }} q^{1 /(2 q-2)}}{\sqrt{m}}\right) p^{1-1 / \phi(m)} .
$$

If $r$ can be chosen less than $p$, then $M_{2}(m, p)<r$. This can be done if

$$
\frac{2^{\phi(m)} \Gamma(\phi(m) / 2+1) \prod_{q \underset{q \mid m}{\operatorname{prime}}} q^{\phi(m) /(2 q-2)}}{m^{\phi(m) / 2}}<p .
$$

\section{A theorem of HeCKE}

The material on the idele group presented here is taken from ([4], pp. 137143, 292-293) and ([1], p. 68). Suppose that $k$ is an algebraic number field with $N=[k: \mathbb{Q}]$, and denote the set of prime ideals in the ring of integers of $k$ by $\mathcal{P}$. Let $\mathcal{M}_{k}$ be the set of absolute values on $k$, where each absolute value generates a different topology on $k$ and is normalized to induce a standard absolute value on $\mathbb{Q}$. A standard absolute value $v$ on $\mathbb{Q}$ is of the form $v(q)=|q|$ or $v(q)=p^{-o_{p}(q)}$, where $p$ is prime and $o_{p}(q)$ is the exponent of $p$ that appears in the prime factorization of $q$. The set of archimedean absolute values is denoted by $S_{\infty}$, and the completion of $k$ with respect to an absolute value $v$ is denoted by $k_{v}$. The archimedean absolute values $v$ on $k$ are all of the form $v(x)=|\sigma(x)|$, where $\sigma$ is an embedding of $k$ into $\mathbb{C}$.

The multiplicative group $k_{v}^{*}$ is locally compact in the topology generated by the absolute value $v$ on $k_{v}$. If $v$ is an absolute value arising from a prime ideal $P$, then the absolute value will be called $P$-adic. If $v$ is a $P$-adic absolute value, then the group $\mathcal{O}_{v}^{*}$ consisting of all $k \in k_{v}^{*}$ with $v(k)=1$ forms a compact open subgroup of $k_{v}^{*}$. This group will be frequently referred to as the $P$-adic units.

If $j \in \prod_{v \in \mathcal{M}_{k}} k_{v}^{*}$, then let $j_{v}$ denote the $v$ th component of $j$. The idele group $J_{k}$ is the set of all $j$ such that $j_{v}$ is a $P$-adic unit for all but finitely many $P$-adic absolute values $v$. The topology on $J_{k}$ is that generated by sets of the form $\prod_{v \in \mathcal{M}_{k}} U_{v}$, where $U_{v}$ is open in $k_{v}^{*}$ and $U_{v}=\mathcal{O}_{v}^{*}$ for all but finitely many $P$-adic valuations $v$. The idele group $J_{k}$ is a locally compact topological group with respect to this topology. 
A number of properties of $J_{k}$ will become useful later on. First of all, note that $\alpha \in k^{*}$ is a $P$-adic unit for all but finitely many $P$-adic absolute values. This implies that $k^{*}$ can be embedded in $J_{k}$ by taking $\alpha$ to $(\alpha, \alpha, \ldots, \alpha, \ldots)$. The quotient $J_{k} / k^{*}$ is called the idele class group of $k$, and is a topological group with the quotient topology.

Secondly, define $\|j\|=\prod_{v \in \mathcal{M}_{k}} v\left(j_{v}\right)$. This product is well-defined, and determines a continuous group homomorphism from $J_{k}$ to the multiplicative group $\mathbb{R}^{+}$ of positive real numbers. The kernel of this map is a closed subgroup of $J_{k}$ denoted by $J_{k}^{0}$. It follows as a consequence of the product formula that $k^{*} \subset J_{k}^{0}$. The projection of $J_{k}^{0}$ to the idele class group gives a compact subgroup $J_{k}^{0} / k^{*}$ (see [4], p. 142).

The multiplicative group $\mathbb{R}^{+}$can be embedded in $J_{k}$ by taking a positive real number $t$ to the idele $j$ whose archimedean components are $t^{1 / N}$ and whose $P$-adic components are 1 . This embedding gives a decomposition of $J_{k}$ as the internal direct product of $J_{k}^{0}$ and $\mathbb{R}^{+}$. Define $J^{S_{\infty}}$ to be the subgroup of $J_{k}$ consisting of all ideles whose archimedean components are 1 and whose $P$-adic components are $P$-adic units. Let $\pi$ be the projection from $J_{k}$ to $J_{k} /\left(\mathbb{R}^{+} k^{*} J^{S_{\infty}}\right)$. The quotient topology induced on $J_{k} /\left(\mathbb{R}^{+} k^{*} J^{S_{\infty}}\right)$ as a quotient of $J_{k}$ is the same as that induced on $J_{k} /\left(\mathbb{R}^{+} k^{*} J^{S_{\infty}}\right)$ as a quotient of the idele class group, and both $\pi$ and the projection $\pi_{2}$ from the idele class group are continuous with respect to this topology. It follows from previous remarks that $\pi\left(J_{k}^{0}\right)=\pi_{2}\left(J_{k}^{0} / k^{*}\right)=J_{k} /\left(\mathbb{R}^{+} k^{*} J^{S_{\infty}}\right)$ is a compact topological group.

If $G$ is a compact topological group, then a character of $G$ is a continuous group homomorphism from $G$ to the unit circle in the complex plane. The definition of equidistribution is given in full generality in ([4], pp. 315-316), but it will only be stated here in the context of prime ideals of the ring of integers of $k$. Define $\tau: \mathcal{P} \rightarrow J_{k}$ as follows. For each prime ideal $P$, select an element $\gamma_{P} \in k_{v_{P}}^{*}$ that generates the prime ideal in $\mathcal{O}_{v_{P}}$, and define $\tau(P)$ to be the idele with $\gamma_{p}$ in the $v_{P}$ th component and 1 in all other components. Let $\mathcal{P}_{r}$ denote the set of prime ideals $P$ such that $N_{k / \mathbb{Q}}(P) \leq r$. If $\lambda$ is a map from $J_{k}$ to a compact commutative group $G$, then $\mathcal{P}$ is $\lambda \circ \tau$-equidistributed in $G$ if

$$
\lim _{r \rightarrow \infty} \frac{1}{\#\left(\mathcal{P}_{r}\right)} \sum_{\psi \in \mathcal{P}_{r}} \chi \circ \lambda \circ \tau(\psi)=\int_{G} \chi
$$

for all characters $\chi$ of $G$. The measure on $G$ is the unique Haar measure $\mu$ with $\mu(G)=1$. The only property of Haar measure that will be used explicitly is that $\mu(g U)=\mu(U)$ for all Borel-measurable sets $U$ and $g \in G$. See [2] for an in-depth exposition of Haar measure.

If $\mathcal{P}$ is $\lambda \circ \tau$-equidistributed in $G$, then equation (2) holds if $\chi$ is replaced by any integrable function on $G$, where an integrable function is as defined in ([4], p. 316). The next section will take for granted the fact that the characteristic function on an open set is integrable.

The following theorem due to Hecke (see [4], p. 317) gives a criterion for $\mathcal{P}$ to be $\lambda \circ \tau$-equidistributed in $G$. It follows from this theorem that $\mathcal{P}$ is $\pi \circ \tau$-equidistributed in $J_{k} /\left(\mathbb{R}^{+} k^{*} J^{S_{\infty}}\right)$.

Theorem 7. If $G$ is a compact commutative group and $\lambda: J_{k} \rightarrow G$ is a continuous homomorphism such that $\lambda\left(J_{k}^{0}\right)=G$ and $\lambda\left(k^{*}\right)=\{1\}$, then $\mathcal{P}$ is $\lambda \circ \tau$-equidistributed in $G$. 
The next section will prove a theorem on the distribution of principal prime ideals in $\mathbb{Z}\left[\zeta_{m}\right]$ where $m$ is a power of 2 or $m=6$.

\section{The Distribution OF PRINCIPAL PRIME IDEALS IN CYCLOTOMIC FIELDS}

The following theorem will be critical in proving that the upper bound derived in Section 2 is optimal if $m$ is a power of 2 or $m=6$.

Theorem 8. If $m$ is a power of 2 or $m=6$, then for all $\epsilon>0$, there is $\gamma=$ $\sum_{j=0}^{\phi(m)-1} \gamma_{j} \zeta_{m}^{j} \in \mathbb{Z}\left[\zeta_{m}\right]$ such that:

1. $\gamma$ generates a prime ideal in $\mathbb{Z}\left[\zeta_{m}\right]$,

2. $N_{\mathbb{Q}\left(\zeta_{m}\right) / \mathbb{Q}}(\gamma)=p$, where $p$ is prime and $p \equiv 1 \bmod m$, and

3. if $\beta=p / \gamma=\sum_{j=0}^{\phi(m)-1} b_{j} \zeta_{m}^{j}$, then $\left|b_{j} / b_{0}\right|<\epsilon$ for $1 \leq j \leq \phi(m)-1$.

There is also a $\gamma$ satisfying the first two conditions together with the condition that $b_{0}>0$ and $1-\epsilon<b_{j} / b_{0}<1+\epsilon$ for $1 \leq j \leq m / 2-1$.

The first step in proving the theorem is to find an open set $U_{\epsilon} \subset J_{k} /\left(\mathbb{R}^{+} k^{*} J^{S_{\infty}}\right)$ with the property that there is a $\gamma$ satisfying conditions 1 and 3 in the theorem if $\pi \circ \tau(P)$ is in $U_{\epsilon}$. The equidistribution criterion together with some additional information on the distribution of primes will then show that there is a $\gamma$ that satisfies all the conditions of the theorem. The proof for the alternate third condition is very similar.

To find $U_{\epsilon}$ if $m$ is a power of 2 , let $\sigma_{0}, \ldots, \sigma_{\phi(m) / 2-1}$ be the embeddings of $k$ into $\mathbb{C}$ defined by $\sigma_{i}\left(\zeta_{m}\right)=\zeta_{m}^{z_{i}}$, where $1=z_{0}<\cdots<z_{\phi(m) / 2-1}=m / 2-1$ are relatively prime to $m$. These embeddings induce all of the archimedean absolute values on $k$, and give a metric $d$ on $k^{\phi(m) / 2}$ defined by $d\left(c, c^{\prime}\right)=\sqrt{\sum_{j=0}^{\phi(m) / 2-1}\left|\sigma_{j}\left(c_{j}-c_{j}^{\prime}\right)\right|^{2}}$, where $c_{j}$ and $c_{j}^{\prime}$ are the $j$ th components of $c_{j}$ and $c_{j}^{\prime}$, respectively. This metric extends to a metric $d$ on $\mathbb{C}^{\phi(m) / 2}$, and $d$ generates the topology on $\mathbb{C}^{\phi(m) / 2}$ as a subset of $J_{k}$. Consider $\mathbb{C}$ as being embedded in $\mathbb{C}^{\phi(m) / 2}$ along the diagonal, and suppose that $c=\sum_{i=0}^{\phi(m)-1} q_{i} \zeta_{m}^{i} \in k^{*}$ with $d(0, c)<\eta / \sqrt{2 \phi(m)}$, where $\eta>0$ is chosen so that $\eta /(1-\eta)<\epsilon$ and $(1-\eta) /(1+\eta)>1-\epsilon$. The following lemma will put a bound on $\left|q_{i}\right|$.

Lemma 8.1. If $c$ is as above, and $m$ is a power of 2 with $m \geq 4$, then $\left|q_{i}\right|<\eta$ for $0 \geq i \geq \phi(m)-1$.

Proof. First of all, note that $j+m / 2$ is relatively prime to $m$ if $j$ is relatively prime to $m$. This implies that $\operatorname{Tr}_{\mathbb{Q}\left(\zeta_{m}\right) / \mathbb{Q}}\left(\zeta_{m}\right)=0$, and hence that $\operatorname{Tr}_{\mathbb{Q}\left(\zeta_{m}\right) / \mathbb{Q}}\left(\zeta_{m}^{i}\right)=0$ if $i \not \equiv 0 \bmod m / 2$, since $\zeta_{m}^{i}$ is a primitive $m / \operatorname{gcd}(m, i)$ th root of unity. It then follows from a straightforward computation that $\operatorname{Tr}_{\mathbb{Q}\left(\zeta_{m}\right) / \mathbb{Q}}\left(c_{j} \overline{c_{j}}\right)=\phi(m) \sum_{i=0}^{\phi(m)-1} q_{i}^{2}$. If $\sigma \in \operatorname{Gal}\left(\mathbb{Q}\left(\zeta_{m}\right) / \mathbb{Q}\right)$, then $\sigma=\sigma_{j}$ or $\sigma=\overline{\sigma_{j}}$ for some $j$. The lemma now follows from the following calculation.

$$
\begin{aligned}
\left|q_{i}\right| & \leq \sqrt{\sum_{j=0}^{\phi(m)-1} q_{i}^{2}} \\
& =\sqrt{\phi(m) \operatorname{Tr}_{\mathbb{Q}\left(\zeta_{m}\right) / \mathbb{Q}}\left(c_{j} \overline{c_{j}}\right)} \\
& =\sqrt{2 \phi(m) d^{2}(0,1 / c)} \\
& <\eta .
\end{aligned}
$$


Let $U=\{c \mid d(c, 1)<\eta / \sqrt{2 \phi(m)}\}$ and let $U_{\epsilon}$ be the open set $\pi\left(U \times \prod_{v} \mathcal{O}_{v}^{*}\right)$, where the product ranges over all $P$-adic absolute values. The process is the same for the alternate third condition, except that $U=\left\{c \mid d\left(c, \sum_{j=0}^{\phi(m)-1} \zeta_{m}^{j}\right)<\eta / \sqrt{2}\right\}$. If $m=6$, then every $c \in \mathbb{C}$ has a unique representation of the form $r_{1}+r_{2} \zeta_{m}$ with $r_{1}, r_{2} \in \mathbb{R}$, and so $U=\left\{c|| r_{1} / r_{2} \mid<\epsilon\right\}$, or $U=\left\{c|| r_{1}-1|<\eta,| r_{2}-1 \mid<\eta\right\}$ for the alternate third condition.

If $\pi \circ \tau(P) \in U_{\epsilon}$, then it is possible to write $\tau(P)=\gamma r j^{S} u$, where $\gamma \in k^{*}, r \in$ $\mathbb{R}^{+}, j^{S} \in J^{S}$, and $u \in U$. An examination of the components shows that $\gamma$ is a prime element in the $P$-adic completion and a $P^{\prime}$-adic unit for all $P^{\prime} \in \mathcal{P}$ that are different from $P$. It follows that $\gamma \in \mathbb{Z}\left[\zeta_{m}\right]$ and that $\gamma$ generates $P$. Since $U$ is an open set, it is possible to find $q \in \mathbb{Q}$ sufficiently close to $r$ so that $1 / \gamma=q u^{\prime}$ with $u^{\prime} \in k^{*} \cap U$. If $u^{\prime}=\sum_{j=0}^{\phi(m)-1} q_{j} \zeta_{m}^{j}$, then $\left|q_{0}-1\right|<\eta$ and $\left|q_{j}\right|<\eta$ for $1 \leq j \leq \phi(m)-1$ by the previous lemma, so that $\left|q_{j}\right| /\left|q_{0}\right|<\epsilon$ for $1 \leq j \leq \phi(m)-1$. This shows that there is a $\gamma$ satisfying conditions 1 and 3 if $\pi \circ \tau(P) \in U_{\epsilon}$. The proof of the equivalent statement for the alternate third condition is the same. Note that it is possible to assume without loss of generality that the $q_{j}$ 's are positive in this case, since the previous lemma shows that they are close to 1 . The same statements follow in a straightforward manner if $m=6$.

If $\chi$ is the characteristic function on $U_{\epsilon}$, meaning that $\chi$ is 1 on $U_{\epsilon}$ and 0 outside of $U_{\epsilon}$, then equation (2) becomes the following equation:

$$
\lim _{r \rightarrow \infty} \frac{\#\left(\mathcal{P}_{r} \cap U_{\epsilon}\right)}{\#\left(\mathcal{P}_{r}\right)}=\mu\left(U_{\epsilon}\right) .
$$

The following lemma will show that there are infinitely many primes $P$ that are in $U_{\epsilon}$.

Lemma 8.2. Suppose $G$ is a compact topological group with the unique Haar measure $\mu$ such that $\mu(G)=1$. If $U$ is a non-empty open subset of $G$, then $\mu(U)>0$.

Proof. If $G$ is a topological group, then $\bigcup_{g \in G} g U$ is an open cover of $G$, and so $G$ can be written in the form $G=\bigcup_{j=0}^{n} g_{j} U$ for some finite set $\left\{g_{0}, \ldots, g_{n}\right\} \subset G$. It follows that $\mu(G)=1 \leq n \mu(U)$ since $\mu$ is Haar measure, and so $\mu(U) \geq 1 / n>0$.

The next lemma will show that there must be infinitely many primes $P$ such that $P \in U_{\epsilon}$ and $P$ satisfies the second condition. This will complete the proof of the theorem.

Lemma 8.3. If $\mathcal{P}^{p}$ is the set of prime ideals with prime norm, then

$$
\lim _{r \rightarrow \infty} \frac{\#\left(\mathcal{P}_{r} \cap \mathcal{P}^{p}\right)}{\#\left(\mathcal{P}_{r}\right)}=1
$$

Proof. If $N_{\mathbb{Q}\left(\zeta_{m}\right) / \mathbb{Q}}(P)=p$ with $p$ prime, then there are $\phi(m)$ prime ideals lying above $p$ and $p \equiv 1 \bmod m$. In general, $N_{\mathbb{Q}\left(\zeta_{m}\right) / \mathbb{Q}}(P)=p^{o(p)}$, and there are $\phi(m) / o(p)$ ideals lying over $p$, where $P \cap \mathbb{Z}=p \mathbb{Z}$, and $o(p)$ is the multiplicative order of $p \bmod m$. The above limit then becomes the following:

$$
\lim _{r \rightarrow \infty} \frac{\phi(m) \#\{p \mid p \text { prime, } p<r, p \equiv 1 \bmod m\}}{\sum_{j \in(\mathbb{Z} / m \mathbb{Z})^{*}} \frac{\phi(m)}{o(j)} \#\left\{p \mid p \text { prime, } p \equiv j \bmod m, p<r^{1 / o(j)}\right\}} .
$$

Divide both the numerator and denominator of this fraction by

$$
\#\{p \mid p \text { prime, } p<r\} \text {. }
$$


By Dirichlet's theorem on the distribution of primes in arithmetic progressions (see [7], p. 31), the numerator and the $1 \bmod m$ component of the denominator tend to 1 as $r \rightarrow \infty$, while the other components of the denominator tend to 0 . To see this fact for the other components, divide the numerator and denominator of the component by $\#\left\{p \mid p\right.$ prime, $\left.p<r^{1 / o(j)}\right\}$. By Dirichlet's theorem, the numerator tends to $1 / o(j)$, and the denominator tends to $r^{1-1 / o(j)} / o(j)$ by the Prime Number Theorem. The lemma now follows.

The next section will be devoted to the application of Theorem 8 to the problem of finding optimal possible bounds for $M_{1}(m, p)$.

\section{What ARE THE OPTIMAL BOUNDS FOR $M_{1}(m, p)$ ?}

If $m$ is a power of 2 , then it was proven in Section 2 that $K_{m} \leq 1$, where $K_{m}$ is as defined in Section 1. The following theorem shows that equality holds.

Theorem 9. If $m$ is a power of 2 , then $K_{m}=1$.

Suppose without loss of generality that $\gamma \mathbb{Z}\left[\zeta_{m}\right]=P_{m-1}$, where $\gamma$ is as in Theorem 8 and $P_{j}$ is as defined in Section 2. It is clear that $\beta=p / \gamma$ is an element of $\mathbb{Z}\left[\zeta_{m}\right]$, and it follows from Theorem 8 that $\left|b_{j} / b_{0}\right|<\epsilon$ for $1 \leq j \leq$ $\phi(m)-1$. The next lemma shows that there is a coset $C$ of $\mu_{m}$ in $(\mathbb{Z} / p \mathbb{Z})^{*}$ with $C=\left\{ \pm b_{0}, \ldots, \pm b_{m / 2-1}\right\}$.

Lemma 9.1. If $m$ is a power of $2, \beta \in \prod_{j=0}^{m / 2-2} P_{2 j+1}$, and $\beta \notin P_{m-1}$, then $b_{i} \equiv$ $b_{0} t^{i} \bmod p$ for $1 \leq i \leq m / 2-1$, and $b_{0} \not \equiv 0 \bmod p$.

Proof. It is a consequence of the definition of $P_{j}$ that $\zeta_{m} \equiv t^{j} \bmod P_{j}$, and hence that $\sum_{i=0}^{m / 2-1} b_{i} t^{i j} \equiv 0 \bmod p$ for all odd numbers $j$ with $j \not \equiv-1 \bmod m$. This means that the vector $v=\left(b_{0} \bmod p, \ldots, b_{m / 2-1} \bmod p\right)$ is in the nullspace of an $(m / 2-1) \times m / 2$ Vandermonde matrix $A$ with nullity $(A)=1$. A geometric series computation shows that $w=\left(1 \bmod p, t \bmod p, \ldots, t^{m / 2-1} \bmod p\right)$ is in the nullspace of $A$, and so $v$ is a scalar multiple of $w$ over $\mathbb{Z} / p \mathbb{Z}$. It follows that $b_{i} \equiv$ $b_{0} t^{i} \bmod p$ for $1 \leq i \leq m / 2-1$, and $b_{0} \not \equiv 0 \bmod p$ since $\beta \notin P_{m-1}$.

Suppose that $C^{\prime}$ is a coset of $\mu_{m}$ in $(\mathbb{Z} / p \mathbb{Z})^{*}$. Let $\beta^{\prime}=\sum_{j=0}^{m / 2-1} b_{j}^{\prime} \zeta_{m}^{j}$, where $b_{0}^{\prime} \in C^{\prime}$ and $b_{j}^{\prime} \equiv b_{0}^{\prime} t^{j}$ for $1 \leq j \leq m / 2-1$. The same argument as in Lemma 1.2 shows that $\beta^{\prime} \in P_{j}$ if $j \neq m-1$, and so $\beta^{\prime}=c \beta$ for some $c=\sum_{j=0}^{m / 2-1} c_{j} \zeta_{m}^{j} \in \mathbb{Z}\left[\zeta_{m}\right]$. Suppose that $\left|c_{k}\right|=\max _{0 \leq j \leq m / 2-1}\left|c_{j}\right|$. The calculation below gives a lower bound for $\left|C^{\prime}\right|$ in terms of $\left|b_{0}\right|$ :

$$
\begin{aligned}
\left|C^{\prime}\right| & \geq\left|b_{k}^{\prime}\right| \\
& =\left|\sum_{j=0}^{k} b_{j} c_{k-j}-\sum_{j=k+1}^{m / 2-1} b_{j} c_{m / 2+k-j}\right| \\
& \geq\left|b_{0} c_{k}\right|-\sum_{j=1}^{k}\left|b_{j} c_{k-j}\right|-\sum_{j=k+1}^{m / 2-1}\left|b_{j} c_{m / 2+k-j}\right| \\
& \geq\left|b_{0} c_{k}\right|(1-(m / 2-1) \epsilon) \\
& \geq\left|b_{0}\right|(1-(m / 2-1) \epsilon) .
\end{aligned}
$$


It also follows that $\left|b_{0}\right| \geq\left(\frac{1}{1+(m / 2-1) \epsilon}\right) p^{1-2 / m}$, since

$$
\begin{aligned}
\left|N_{\mathbb{Q}\left(\zeta_{m}\right) / \mathbb{Q}}(\beta)\right| & =p^{m / 2-1} \\
& =\prod_{j=0}^{m / 2-1}\left|\sum_{l=0}^{m / 2-1} b_{l} \zeta_{m}^{l(2 j+1)}\right| \\
& \leq \prod_{j=0}^{m / 2-1} \sum_{l=0}^{m / 2-1}\left|b_{l}\right| \\
& \leq\left(\left|b_{0}\right|(1+(m / 2-1) \epsilon)\right)^{m / 2} .
\end{aligned}
$$

Combining the inequalities for $\left|C^{\prime}\right|$ and $\left|b_{0}\right|$ gives

$$
M_{1}(m, p) \geq\left(\frac{1-(m / 2-1) \epsilon}{1+(m / 2-1) \epsilon}\right) p^{1-2 / m} .
$$

Letting $\epsilon \rightarrow 0$ proves Theorem 9 .

Let $\kappa_{m}$ be the supremum of all $\kappa$ such that $M_{1}(m, p) \geq \kappa p^{1-1 / \phi(m)}$ for all primes $p$. Section 2 gives a lower bound for $\kappa_{m}$, and the following theorem applies Theorem 8 to give an upper bound in the case where $m$ is a power of 2 . This theorem proves that the upper bound $M_{1}(4, p) \geq(\sqrt{2} / 2) p^{1 / 2}$ proven in Section 2 is optimal.

Theorem 10. If $m$ is a power of 2 , then $\kappa_{m} \leq \frac{1}{2^{1-2 / m}}$.

Proof. Let $\beta$ satisfy the alternate third condition in Theorem 8 . Write $\beta=$ $b_{0}\left(\sum_{j=0}^{m / 2-1} \zeta_{m}^{j}+\sum_{j=0}^{m / 2-1} d_{j} \zeta_{m}^{j}\right)$, where $\left|d_{j}\right|<\epsilon$ for $0 \leq j \leq m / 2-1$, and note that $\sum_{j=0}^{m / 2-1} \zeta_{m}^{j}=2 /\left(1-\zeta_{m}\right)$ with $N_{\mathbb{Q}\left(\zeta_{m}\right) / \mathbb{Q}}\left(2 /\left(1-\zeta_{m}\right)\right)=2^{m / 2-1}$. An upper bound will be placed on $\left|b_{0}\right|$ by using the fact that $p^{m / 2-1}=\left|N_{\mathbb{Q}\left(\zeta_{m}\right) / \mathbb{Q}}(\beta)\right|$. The right-hand side of this equality expands as

$$
\left|b_{0}\right|^{m / 2}\left|\prod_{i=0}^{m / 2-1} 2 /\left(1-\zeta_{m}^{2 i+1}\right)+\sum_{j=0}^{m / 2-1} c_{j} \zeta_{m}^{(2 i+1) j}\right| .
$$

One term in the product is $N_{\mathbb{Q}\left(\zeta_{m}\right) / \mathbb{Q}}\left(2 /\left(1-\zeta_{m}\right)\right)$, and each of the other $2^{m / 2}-1$ terms has absolute value that is bounded above by

$$
f(m) \epsilon=(1 /(1-\cos (2 \pi / m)))^{m / 2-1} m \epsilon .
$$

The cosine term comes from the absolute value of $\left|1-\zeta_{m}\right|$ and the $m \epsilon$ term is an upper bound for $\sum_{j=0}^{m / 2-1} c_{j} \zeta_{m}^{i j}$. Putting these bounds together gives the inequality

$$
p^{m / 2-1} \geq\left|b_{0}\right|^{m / 2}\left|2^{m / 2-1}-\left(2^{m / 2}-1\right) f(m) \epsilon\right|,
$$

or

$$
\left|b_{0}\right| \leq\left(\frac{1}{\left|2^{m / 2-1}-\left(2^{m / 2}-1\right) f(m) \epsilon\right|^{2 / m}}\right) p^{1-2 / m}
$$

Lemma 9.1 shows that there is a $\operatorname{coset} C$ with $C=\left\{ \pm b_{0}, \ldots, \pm b_{m / 2-1}\right\}$, and so $M_{1}(m, p) \leq|C| \leq(1+\epsilon)\left|b_{0}\right|$. Letting $\epsilon \rightarrow 0$ proves the theorem.

If $m=2^{e} 3^{f}$ with $e, f>0$, then the upper bound

$$
M_{1}(m, p) \leq(2 / \sqrt{3}) p^{1-m / 3}
$$


proven in Section 2 is the same as the bound proven by Robinson. This suggests that perhaps this bound is optimal. In general, this seems difficult to prove, in part because it seems difficult to extend Theorem 8 to this case. Theorem 8 does apply, however, when $m=6$, and plays a crucial role in the following result, which shows that the upper bound from Section 2 is optimal.

Theorem 11. If $K_{m}$ and $\kappa_{m}$ are as previously defined, then $K_{6}=2 / \sqrt{3}$ and $\kappa_{6} \leq 1$.

Proof. Pick $\beta$ and $\gamma$ that satisfy the alternate third condition in Theorem 8 , and suppose without loss of generality that $\gamma \mathbb{Z}\left[\zeta_{m}\right]=P_{m-1}$, so that $\beta \mathbb{Z}\left[\zeta_{m}\right]=P_{1}$. It follows that $b_{0}+b_{1} t \equiv 0 \bmod p$, and hence that $b_{1} \equiv b_{0} t^{2} \bmod p$. This means that $b_{0}+b_{1} \equiv b_{0}\left(1+t^{2}\right) \equiv b_{0} t \bmod p$, which gives a coset $C=\left\{ \pm b_{0}, \pm\left(b_{0}+b_{1}\right), \pm b_{1}\right\}$. Note that

$$
N_{\mathbb{Q}\left(\zeta_{m}\right) / \mathbb{Q}}(\beta)=p=b_{0}+b_{0} b_{1}+b_{1}^{2} \leq b_{0}^{2}\left(1+(1+\epsilon)+(1+\epsilon)^{2}\right),
$$

which implies that $b_{0}+b_{1} \geq(2-\epsilon)\left(\frac{p}{1+(1+\epsilon)+(1+\epsilon)^{2}}\right)^{1 / 2}$.

Suppose that $C^{\prime}$ is another coset with $C^{\prime}=\left\{ \pm b_{0}^{\prime}, \pm\left(b_{0}^{\prime}+b_{1}^{\prime}\right), \pm b_{1}^{\prime}\right\}$, where $b_{1} \equiv$ $b_{0} t^{2} \bmod p$. It follows that $b_{0}^{\prime}+b_{1}^{\prime} \zeta_{m} \in P_{1}$, and so

$$
b_{0}^{\prime}+b_{1}^{\prime} \zeta_{m}=\left(c_{0}+c_{1} \zeta_{m}\right)\left(b_{0}+b_{1} \zeta_{m}\right)
$$

for some $c_{0}, c_{1} \in \mathbb{Z}$. If $c_{1}=0$, then $\left|b_{0}^{\prime}+b_{1}^{\prime}\right|=\left|c_{0}\right|\left|b_{0}+b_{1}\right| \geq\left|b_{0}+b_{1}\right|$. Suppose now without loss of generality that $c_{1}>0$. If $c_{0}=0$, then $\left|b_{1}^{\prime}\right|=c_{1}\left|b_{0}+b_{1}\right|$. If $c_{0}>0$, then $\left|b_{1}^{\prime}\right|=\left(c_{0}+c_{1}\right) b_{1}+c_{1} b_{0}>b_{0}+b_{1}$, and $c_{0}<0$ implies that $\left|b_{0}^{\prime}\right|=\left|c_{0} b_{0}-c_{1} b_{1}\right|>b_{0}+b_{1}$. It now follows that

$$
\left|C^{\prime}\right| \geq|C| \geq(2-\epsilon)\left(\frac{p}{1+(1+\epsilon)+(1+\epsilon)^{2}}\right)^{1 / 2},
$$

which proves the first part of the theorem.

To prove the second part of the theorem, let $\gamma, \beta$, and $C$ be as before, except that $\beta$ satisfies the regular third condition of Theorem 8 . Under these circumstances, it follows that $|C| \leq(1+\epsilon)\left|b_{0}\right|$ and that

$$
p=b_{0}^{2}+b_{0} b_{1}+b_{1}^{2} \leq b_{0}^{2}\left(1+\epsilon+\epsilon^{2}\right) .
$$

Solving for $b_{0}$ gives $|C| \leq \frac{1+\epsilon}{\sqrt{1+\epsilon+\epsilon^{2}}} \sqrt{p}$, which proves the second part of the theorem.

In general, the bounds from Section 2 can be improved by finding the volume of $S_{1 / 2} \cap V$ exactly instead of using the Vaaler estimate. It is doubtful, however, that this improvement will lead to an optimal bound.

\section{ACKNOWLEDGMENT}

The author would like to thank Hendrik Lenstra for his insights and suggestions, and would like to acknowledge the late Rafael Robinson, whose work forms the basis for this paper.

\section{REFERENCES}

1. J.W.S. Cassels and A. Frohlich, Algebraic Number Theory, Academic Press Limited, 1967. MR 35:6500

2. John L. Kelley and T.P. Srinivasan, Measure and Integral, Springer-Verlag, 1988. MR 89e:28001 
3. Sergey Konyagin and Igor Shparlinski, On the Distribution of Residues of Finitely Generated Multiplicative Groups and Some of Their Applications, to appear.

4. S. Lang, Algebraic Number Theory, Springer-Verlag, 1994. MR 95f:11085

5. C.G. Lekkerkerker, Geometry of Numbers, Wolters-Noordhoff and North-Holland Publishing Companies, 1969. MR 42:5915

6. R.M. Robinson, Numbers Having $m$ Small mth Roots $\bmod p$, Mathematics of Computation 61 (1993), no. 203, 393-413. MR 93k:11002

7. P. Stevenhagen and H.W. Lenstra, Jr., Chebotarev and his density theorem, Math. Intelligencer 18 (1996), 26-37. CMP 96:14

8. J.E. Vaaler, A Geometric Inequality with Applications to Linear Forms, Pacific Journal of Mathematics 83 (1979), no. 2, 543-553. MR 81d:52007

Department of Mathematics, University of California at Berkeley, Berkeley, CalIFORNIA 94720 